Research papers

\title{
Liver MRI: From basic protocol to advanced techniques
}

\author{
Henrique Donato $^{\mathrm{a}, *}$, Manuela França ${ }^{\mathrm{b}}$, Isabel Candelária ${ }^{\mathrm{a}}$, Filipe Caseiro-Alves ${ }^{\mathrm{a}}$ \\ a Imaging Department, Faculty of Medicine of Coimbra, University Centre Hospitals of Coimbra (CHUC), Portugal \\ b Imaging Department, Centro Hospitalar do Porto, Portugal
}

\section{A R T I C L E I N F O}

\section{Keywords:}

MRI

Liver

Diffusion MRI

Perfusion imaging

Fatty liver

Liver cirrhosis

\begin{abstract}
A B S T R A C T
Liver MR is a well-established modality with multiparametric capabilities. However, to take advantage of its full capacity, it is mandatory to master the technique and optimize imaging protocols, apply advanced imaging concepts and understand the use of different contrast media. Physiologic artefacts although inherent to upper abdominal studies can be minimized using triggering techniques and new strategies for motion control. For standardization, the liver MR protocol should include motion-resistant T2-w sequences, in-op phase GRE T1 and T2-w fast spin echo sequences with fat suppression. Diffusion-weighted imaging (DWI) is mandatory, especially for detection of sub-centimetre metastases. Contrast-enhanced MR is the cornerstone of liver MR, especially for lesion characterization. Although extracellular agents are the most extensively used contrast agents, hepatobiliary contrast media can provide an extra-layer of functional diagnostic information adding to the diagnostic value of liver MR. The use of high field strength (3T) increases SNR but is more challenging especially concerning artefact control. Quantitative MR belongs to the new and evolving field of radiomics where the use of emerging biomarkers such as perfusion or DWI can derive new information regarding disease detection, prognostication and evaluation of tumour response. This information can overcome some of the limitations of current tests, especially when using vascular disruptive agents for oncologic treatment assessment. MR is, today, a robust, mature, multiparametric imaging modality where clinical applications have greatly expanded from morphology to advanced imaging. This new concept should be acknowledged by all those involved in producing high quality, high-end liver MR studies.
\end{abstract}

\section{Introduction}

MR is a well-established liver imaging modality that has been subject to continuous improvement, through advances in hardware, software and contrast agent development $[1,2]$. It provides a comprehensive assessment of tissue characteristics through its multiparametric capabilities, providing accurate qualitative and quantitative data $[3,4]$.

When compared to its main competitor CT, MR has a higher contrast-to-noise ratio (CNR), lack of ionizing radiation exposure and uses contrast agents with the ability to explore both extracellular and hepatocellular compartments [2,3,5].

There are however some constraints, like higher cost, longer acquisition time, greater need for patient collaboration and individual patient limitations, like claustrophobia, presence of pacemakers and poor renal function, the latter specifically for contrast-enhanced MR (DCE-MR) [5].

In order to use the abilities of liver MR to its full extent, performing high quality efficient exams, it is mandatory to use the best imaging protocol, to optimize the technique, to minimize artefacts and to select the most adequate type of contrast agent [4].

\subsection{Patient preparation and positioning}

Patients should receive general instructions regarding the magnetic field, highlighting the importance of immobilization throughout the entire exam and the need for a shallow, regular breathing motion, crucial for free-breathing and respiratory-triggering techniques $[6,7]$. Breath-hold acquisitions should also be explained at the beginning of the exam [6]. Placing the patient in a comfortable supine position, which may include knee support by a foam pad, is important for immobilization and compliance to other instructions [6,7]. Placing an abdominal cushion may be useful to minimize dielectric effect observed on 3T magnets $[8,9]$.

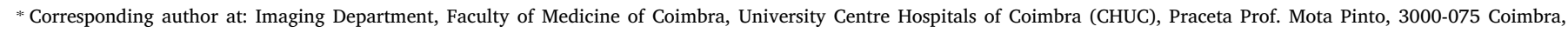
Portugal/Present address: Quinta de Voimarães, lote 7, $7^{\circ}$ esq, 3000-377 Coimbra, Portugal.

E-mail addresses: donato.henrique@gmail.com (H. Donato), mariamanuela.franca@gmail.com (M. França), auxilio.mate@gmail.com (I. Candelária), caseiroalves@gmail.com (F. Caseiro-Alves). 
Table 1

Sequence parameters for $1.5 \mathrm{~T}$.

\begin{tabular}{|c|c|c|c|c|c|c|c|c|c|}
\hline Sequence & Plane & TR (ms) & TE (ms) & Flip angle & Fat saturation & Matrix & FOV $(\mathrm{mm})$ & No. slices & Slice thickness \\
\hline HASTE & coronal & 900 & 77 & $150^{\circ}$ & none & $256 \times 243$ & 400 & 40 & $5 \mathrm{~mm}$ \\
\hline HASTE & axial & 900 & 77 & $150^{\circ}$ & none & $256 \times 218$ & 400 & 44 & $5 \mathrm{~mm}$ \\
\hline T2 TSE FS & axial & 1550 & 93 & $150^{\circ}$ & Fat sat & $384 \times 269$ & 380 & 20 & $8 \mathrm{~mm}$ \\
\hline T1 GRE in and out of phase & axial & 100 & $\begin{array}{l}2.27 \\
5.19\end{array}$ & $70^{\circ}$ & none & $256 \times 192$ & 380 & 20 & $8 \mathrm{~mm}$ \\
\hline Diffusion & axial & 2300 & 70 & $90^{\circ}$ & Fat sat & $160 \times 120$ & 450 & 20 & $8 \mathrm{~mm}$ \\
\hline T1 3D GRE VIBE & axial & 4.88 & 2.38 & $10^{\circ}$ & Fat sat & $256 \times 205$ & 380 & 60 & $3 \mathrm{~mm}$ \\
\hline T1 3D GRE VIBE hepatobiliary & axial & 4.88 & 2.38 & $30^{\circ}$ & Fat sat & $256 \times 205$ & 380 & 60 & $3 \mathrm{~mm}$ \\
\hline
\end{tabular}

\section{Standard liver protocols}

An adequate MRI protocol has to be short, comprehensive and standardized to allow reproducibility and consistency of image quality and diagnostic information. It allows evaluation of the liver parenchyma, vasculature and biliary system [2]. Examples of sequence parameters are displayed in Table 1.

\subsection{Coil design}

A body or torso phased array coil is available in almost all manufactures and should be used for all sequences [4,6]. This type of coil enhances signal-to-noise ratio (SNR), including the subcutaneous fat signal, accentuating motion artefacts. To minimize these artefacts, free-breathing sequences should be performed with fat suppression. Phased array coils also permit the use of parallel imaging, which improves scan time or resolution [6]. In obese patients, adequate coil positioning can be checked by a scout sequence and repositioned if needed [7].

\subsection{Imaging planes}

Single shot fast spin echo (SSFSE) images, performed at the beginning of the exam, are obtained in the coronal and axial planes $[4,10]$. They provide a valuable initial overview of the liver and upper abdomen [6]. The remaining sequences of the standard liver protocol are performed solely in the axial plane $[4,10]$. However, additional coronal or sagittal images can be obtained when investigation of anatomical relationships so warrants [6].

\subsection{Artefact control}

Despite all developments, MR diagnostic performance is still affected by artefacts, especially those caused by motion [2,7]. Measures can be taken to avoid voluntary movements but physiologic motion is inherent to any liver imaging protocol, whether it results from breathing or cardiac motion, blood flow and vessel pulsation or even gastrointestinal peristalsis $[6,11,12]$. Movement during image acquisition leads to blurring and ghosting, image duplicates from misplaced signal that may hamper image interpretation (Fig. 1) [6,7]. Strategies to reduce motion artefacts include signal averaging; ultrafast motionresistant sequences, like HASTE, FLASH or TFE; and ubiquitous use of fat suppression [6].

Breath hold, free breathing and respiratory triggering techniques are used to control breathing motion, the last two leading to an increase in scan time. The use of navigator echoes for respiratory triggering is among the most popular and well succeeded techniques $[4,6,12]$.

Cardiac motion affects mostly the left liver lobe and can be overcome by ECG or pulse triggering techniques, but again with an acquisition time penalty [12].

To avoid blood flow artefacts, presaturation bands and flow compensation techniques are the main strategies. Presaturation bands are applied to the vessels above and below the target region and are

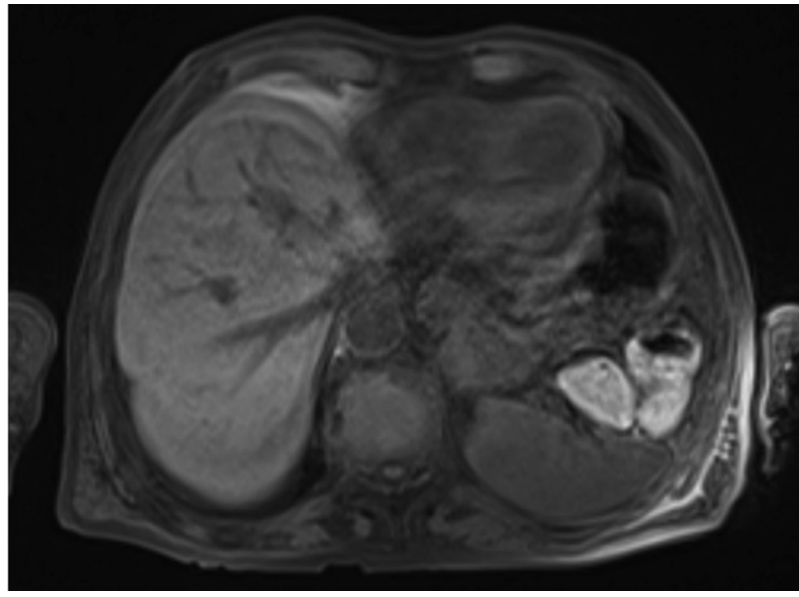

Fig. 1. T1-w fat sat sequence before intravenous contrast administration showing examples of cardiac and respiratory motion artefacts, producing multiple ghost images that reduce the diagnostic information.

especially important for T1-weighted (T1-w) gradient echo (GRE) sequences, like those used for the dynamic study. Flow compensation or gradient moment nulling techniques only correct constant flow. Since they lead to an increase in echo time (TE), they are mainly applied to T2-weighted (T2-w) sequences. The resulting brighter blood vessels in the liver may mimic focal lesions when seen in cross section $[6,7]$.

Antispasmodic agents reduce motion artefacts from gastrointestinal peristalsis, but they are not usually required for liver MR [6].

The standard use of multichannel, multielement phased array coils has allowed the use of parallel imaging technique, which has dramatically improved SNR, accelerating the k-space acquisition, reducing scan times and susceptibility artefacts. Acceleration factors, or the number of lines of k-space acquired in parallel, are typically limited by the development of residual artefacts and severe signal loss, thus, a factor higher than 2 is rarely used [7,11,12]. Acronyms of parallel imaging techniques include GRAPPA, SENSE and mSENSE [11].

Wraparound artefacts can occur when the field of view is too small regarding the size of the abdomen. They may occur in 3D techniques in the slice direction or the phase encoding direction. These artefacts can be corrected by increasing the field of view, using pre-saturation bands or using phase oversampling, although this last choice increases the in scan time [4,7].

Field inhomogeneity caused by magnetic susceptibility due to air or metal leads to local signal loss, distortion and inhomogeneous fat suppression. GRE sequences are especially sensitive and decreasing TE can assist in reducing these artefacts [7].

\subsection{Basic sequences}

\subsubsection{SSFSE}

Single Shot Fast Spin Echo (SSFSE) images are acquired in the beginning of the liver MR protocol (Fig. 2A). Many authors recommend 


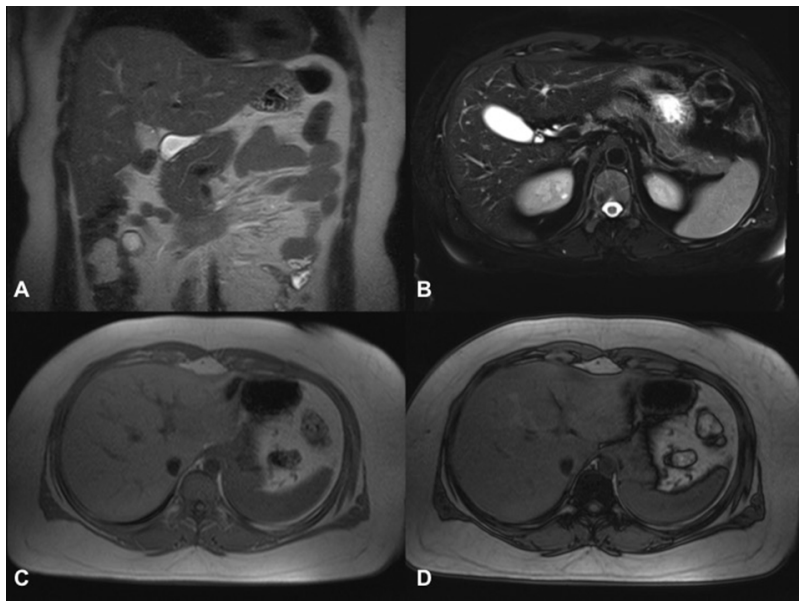

Fig. 2. A. HASTE sequence in the coronal plane. B. T2-w FS sequence. C,D. T1-w sequence in phase and opposed phase, showing in this case loss of signal of the liver parenchyma in the opposed phase acquisition (D), indicating fatty infiltration.

heavily T2-w images, with a TE greater than $160 \mathrm{~ms}$, ideally 180-200 ms. These longer TEs can be useful for differentiating cysts and hemangiomas from solid liver tumours $[4,6,10]$. The sequence has a single excitation pulse, followed by a long series of $180^{\circ}$ refocusing pulses generating echo trains. It can be further accelerated by the acquisition of half of the $\mathrm{k}$ space (HASTE sequence). These sequences have consequently a greater resistance to susceptibility and motion artefacts, since it takes one second or less to obtain each slice and one or two breath-holds for the entire acquisition $[4,13]$. If two breath-holds are required, a sequential acquisition should be performed to avoid misregistration artefacts [4].

Soft tissue detail is generally blurry and there is considerable T2 decay from the long echo train [13]. Fat saturation (FS) is not recommended for this sequence, since it obscures liver margins and reduces the already relatively low overall signal [4].

\subsection{2. $T 1$}

T1 sequences allow detection of fat and other substances with high T1 signal, such as haemorrhage, high protein content and deposition of copper or glycogen $[2,4,14]$. On the other hand, fluid, a cellular or fibrotic content display a low signal on T1-w sequences [2].

GRE are generally used. These sequences are very sensitive to susceptibility artefacts and therefore may assist in the detection of iron, calcium, air or metal. Currently they are used to obtain a dual echo pair of images, exploring the cancelation effects of coexistent fat/water molecules within the same imaging voxel. The TE should be the shortest possible to reduce the $\mathrm{T} 2 *$ decay $[2,4]$. To prevent ambiguity from fatwater interference or susceptibility, the out-phase TE should be lower than the in-phase TE, usually $2.3 \mathrm{~ms}$ for out-phase and $4.6 \mathrm{~ms}$ for inphase imaging at $1.5 \mathrm{~T}[4,8]$. These sequences allow the detection of intracellular fat, either in hepatic lesions or in the hepatic parenchyma [2]. In the out-phase sequence, interfaces between tissues of different resonance frequencies (like pure fat and water) appear dark, creating the so called india-ink artefact (Fig. 2C,D) [6,8].

3D sequences are increasingly being used, obtained with a modified Dixon technique, which explores the chemical shift effect of water and fat, allowing to separate water and fat peaks contributing to better image homogeneity, fat-cancelation, also providing thinner slice thickness and slice overlap [4]. 3D GRE T1-w FS sequences (e.g. LAVA, VIBE, THRIVE) are the typical sequences used for the dynamic contrastenhanced study $[2,4]$.

\subsection{3. $T 2$}

Fast/turbo spin echo (FSE/TSE) T2-w sequences are generally used, with a repetition time (TR) in the range of $2500 \mathrm{~ms}$ and a TE of
$60-120 \mathrm{~ms}$, ideally $80-100 \mathrm{~ms}$, leading to moderate T2 weighting $[2,4,6]$. Since FSE T2-w sequences suffer from magnetization transfer effects that maintain the high signal intensity from abdominal fat, fat suppression should be regularly applied (Fig. 2B) $[6,13]$. Fluid content is the predominant information retrieved from this type of sequence, allowing the distinction of solid from cystic-like focal lesions [2,4]. The signal of the spleen should be used as the internal comparative signal intensity since the majority of malignant lesions tend to display a similar signal intensity [4].

Obtaining the T2 FS images after contrast administration is not only possible but may even be advantageous, since the T2 shortening effects of gadolinium, reduces the baseline T2 signal intensity of the liver [4].

Short time inversion recovery sequences (STIR) are an alternative to FSE T2 FS sequences. Focal lesions will display a higher CNR due to profound fat signal suppression when the appropriate inversion time is used (around $150 \mathrm{~ms}$ at $1.5 \mathrm{~T}$ ) and does not dependent on the fat suppression homogeneity. As drawbacks they need longer acquisition time, and possess lower SNR and less specific suppression, including for blood or gadolinium-enhanced tissues. Therefore, STIR sequences should not be obtained after contrast $[4,6]$.

\subsection{Diffusion weighted imaging}

Diffusion is a physical process of random movement of water molecules. This movement is restricted by cell membranes, resulting in tissues with different cellularity having differences in water mobility [2,12]. A specialized MR tool, diffusion weighted imaging (DWI) derives image contrast from these differences [5,11]. Highly cellular tissues, like neoplasms, show restriction to diffusion and appear hyperintense in DW images [2,12].

DWI has been increasingly applied to liver MR as an excellent complementary tool for lesion detection and characterization, increasing clinical confidence and decreasing false positives $[5,11,14]$.

Oncology is a major application of DWI, with a particular value in the detection of liver metastases, especially sub-centimetre lesions $[11,12,14]$. The role of DWI in distinguishing solid benign liver lesions from malignant ones is more limited and generally requires additional input from DCE-MR $[5,14]$. DWI may also help in predicting and monitoring tumour response to treatment $[5,12]$.

The interpretation of DW images can be done visually and quantitatively, through the apparent diffusion coefficient (ADC) map [12,14].

Eco Planar Imaging (EPI) sequences are widely used for DWI, which are basically T2-w images, acquired with single shot technique and FS. They are essential to rapidly capture the diffusion signal before it becomes null while also being relatively insensitive to patient motion $[6,11,12]$. The TR should be greater than $2500 \mathrm{~ms}$, at least 3 times the T1 of a typical metastasis, to minimize T1 saturation effects and improve accuracy of ADC calculations. Image quality deteriorates rapidly as TE increases, so it should be reduced, by decreasing the acquisition matrix, which ends up to be lower than other sequences, generally in the range of $128 \times 128$ [4].

Different series of DW images are acquired through modification of the gradient strength and magnitude, referred as b-value (Fig. 3) [11].

One series should be obtained with a b-value of 0 , meaning no gradient is applied and consequently no diffusion information is retrieved, giving similar information as T2 FS sequences $[4,11]$.

Another series should be obtained with a low b-value $(b<100)$. These are valuable for lesion detection, since there is a "black blood" effect, that improves conspicuity of lesions located near the dark vessels $[4,11,12]$. Relatively to higher b-value images, they present greater SNR and CNR and are less affected by artefacts [12].

Series obtained with a high b-value (such as $b=800$ ) are important for liver lesion characterization [4]. In clinical practice, b-values higher than $1000 \mathrm{~s} / \mathrm{mm}^{2}$ are seldom used due to the relatively short T2relaxation time of the normal liver parenchyma [15].

EPI sequences are very sensitive to magnetic field inhomogeneity 


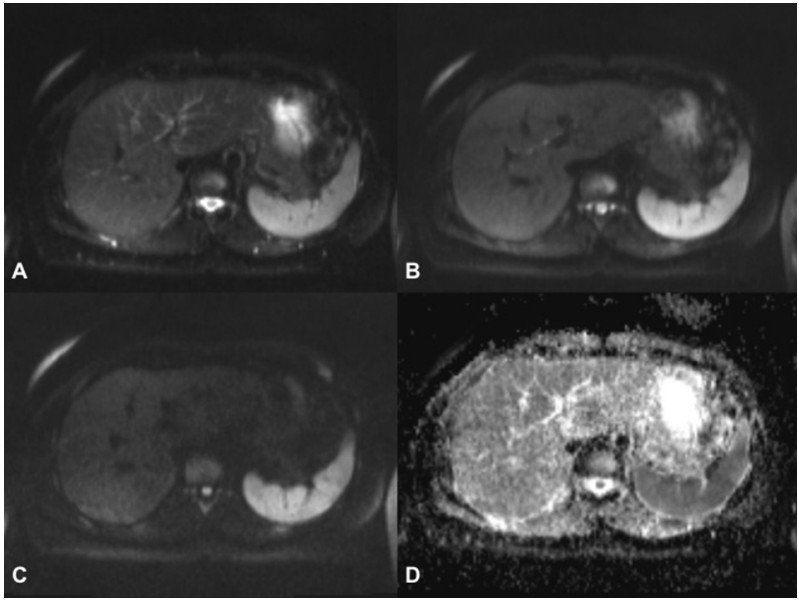

Fig. 3. DW images acquired without gradient application $(A ; b$ value $=0)$, with a low $b$ value $(B ; b=50)$ and with a high $b$ value $(C ; b=700)$. $D-A D C$ map.

and susceptibility artefacts, which lead to image degradation and distortion. Causes include air-tissue interfaces, metal implants and Nyquist ghosting from eddy currents produced by the rapidly switching gradients $[11,12]$. SNR can be improved by increasing the field strength and by signal averaging [11].

The ADC map is the graphical representation of the ratio of DW signal intensities and its measurements may discriminate between benign and malignant lesions $[12,14]$. There may be also potential roles in tumour grading and liver fibrosis assessment $[3,15]$. The ADC measurements are affected by the sequence acquisition protocol and suffer from a lack of reproducibility, particularly in respiratory triggering techniques, left liver lobe seated lesions, smaller size and lesion heterogeneity $[11,12,15]$. Accurate estimation of ADC can be improved by acquiring a large number of b-values, taking into consideration the diffusion profile of the specific tissue, which might be multiexponential [11].

\subsection{Contrast media administration}

Appropriate detection and characterization of liver lesions requires contrast-enhanced imaging, included in most liver MR protocols and generally regarded as the most important sequences $[4,10,14,16]$.

MR contrast agents in current clinical use are composed of gadolinium (Gd) chelates [16]. These agents markedly shorten the T1 relaxation times and their effects are observed in heavily $\mathrm{T} 1-\mathrm{W}$ sequences [6]. Three-dimensional FS GRE T1-w sequences are the basis for MR dynamic analysis, obtained before and through successive phases after intravenous contrast administration $[2,4]$. These sequences have sufficient temporal resolution to be performed in a single breath hold, with good spatial resolution and SNR. Parallel imaging allows an increase in spatial resolution or alternatively a decrease in acquisition time [17].

The TR and TE of these sequences should be as short as possible. A short TR allows for reduction in acquisition time and an increase in T1weighting, while a short TE minimizes susceptibility artefacts. Flip angles typically vary between 10 and $15^{\circ}$.

Fat suppression is fundamental to improve visualization of liver lesions, reducing the abdominal wall motion artefacts [4].

\subsubsection{Extra-cellular agents}

These are the best documented and most extensively used contrast agents in liver MR $[5,14]$. They rapidly distribute throughout the extracellular space and are excreted by glomerular filtration, in a manner similar to CT iodinated-contrast agents, allowing multi-phase dynamic imaging $[2,4,5]$. In comparison to CT contrast agents, they exhibit better enhancement of the blood pool on equilibrium phase and

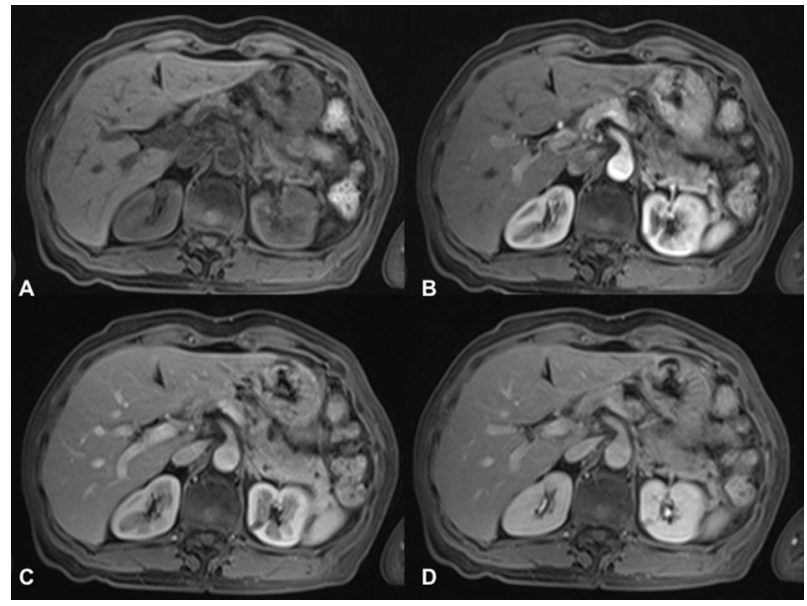

Fig. 4. T1 FS (VIBE) sequences before (A) and after intravenous extracellular Gd-based contrast agent in the arterial phase (B), portal phase (C) and late venous phase (D).

better delineation of subtle areas of contrast accumulation due to a paramagnetic amplification effect [10].

There are several formulations of extra-cellular Gd agents with essentially the same pharmacologic and imaging characteristics [10]. About $10-20 \mathrm{~mL}$ of contrast are generally injected, with a dose of $0.1 \mathrm{mmol} / \mathrm{Kg}$ and an ideal flow rate of $2 \mathrm{~mL} / \mathrm{s}$. Gadobutrol has twice the concentration of other agents so it can be injected at $1 \mathrm{~mL} / \mathrm{s}$ or previously diluted. A $20 \mathrm{~mL}$ saline flush after contrast injection is recommended $[6,16,18]$.

Precontrast images are important to characterize intrinsic lesion properties (such as the presence of paramagnetic substances), and for baseline comparison with postcontrast acquisitions and to assess the technical quality before the dynamic imaging (Fig. 4A) [4,16]. The late arterial phase, occurring $30 \mathrm{~s}$ after intravenous contrast injection, is important for lesion and vascular assessment (Fig. 4B) [16,19]. Signs of an adequate phase include presence of contrast in the hepatic artery and early enhancement of the portal vein, with no or minimal liver parenchymal enhancement $[2,16]$. To ensure adequate timing, a fixed delay or an individual delay can be applied, either with a test bolus or using bolus tracking (CARE bolus software) [2]. The portal venous phase, occurring 60-70 s after the injection, is important for detection of hypovascular lesions and for characterization, looking up for washout (Fig. 4C) [16,19]. A correct portal phase is characterized by enhancement of the entire hepatic vasculature as well as strong liver parenchymal enhancement $[2,16]$. The late venous or interstitial phase is routinely performed in liver MR exams, acquired between 3 and 8 min after contrast media administration (Fig. 4D). This phase has better depiction of the washout sign, and is also important for the assessment of persistent enhancement of hemangiomas and delayed enhancement of fibrotic components in tumours, like cholangiocarcinoma $[2,5,16]$.

\subsubsection{Hepato-biliary agents}

These agents possess a variable degree of hepatic uptake and biliary excretion, enabling the acquisition of an hepatobiliary phase which may overcome some extracellular agents limitations and add diagnostic value (Fig. 5) [2,10,16,19]. They offer improved lesion detection and characterization, as well as functional and biliary assessment $[1,5,10,14]$. These Gd-based agents are biphasic agents, with both an initial distribution phase into the extracellular space and a later hepatobiliary phase $[10,16,20]$. The uptake and biliary excretion are mediated by active transportation through cellular membrane transporters in the hepatocytes, described as OATP B1/3 and MRP2 [1,2]. This only occurs in normal functioning hepatocytes, so hepatocellular lesions conserving those properties will show T1-w enhancement in the hepatobiliary phase, distinct from lesions lacking membrane receptors 


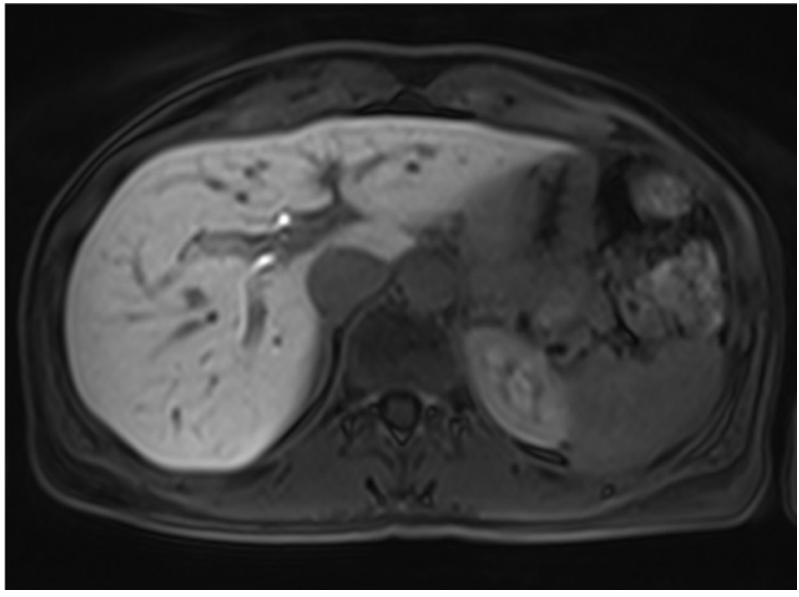

Fig. 5. T1 FS (VIBE) sequence after intravenous Gd-EOB-DTPA administration in the hepatobiliary phase, with enhancement of the liver parenchyma and contrast in the biliary tree.

that appear hypointense compared to the normal parenchyma $[2,5,10,16]$.

There are two Gd-based hepato-specific agents, gadobenate dimeglumine (Gd-BOPTA, Multihance , Bracco Imaging, Milan, Italy) and gadoxetic acid (Gd-EOB-DTPA, Primovist ${ }^{\circledR}$, Bayer Schering Pharma AG, Berlin, Germany), with different profiles [14].

Gd-BOPTA has a recommended dose of administration of $0.1 \mathrm{mmol} /$ $\mathrm{Kg}$ and about $5 \%$ of the dose is excreted through the biliary tract $[1,14,16,20]$. This agent has a better dynamic profile than Gd-EOBDTPA, with higher enhancement of hepatic vascular structures $[1,2,18]$. Hence, some authors prefer it when dynamic characterization is more important $[10,14]$. The hepatobiliary phase is obtained $1-2 \mathrm{~h}$ after administration and therefore does not interfere with washout assessment [16].

On the other hand, Gd-EOB-DTPA has a recommended dose of $0.025 \mathrm{mmol} / \mathrm{Kg}$ and about $50 \%$ of the dose is excreted through the biliary tract $[1,14,16]$. There is rapid uptake and the hepatobiliary phase is obtained just 20 min after administration, when maximum parenchymal enhancement is seen $[14,16,20]$. There is growing evidence that in normal functioning livers a 10 min delay may suffice $[1,19]$. Considering its pharmacodynamic profile there is no true interstitial phase but a transitional phase in which cellular contrast retention already contributes to lesion enhancement [18]. The vascular enhancement is lower and shorter in duration, compared to Gd-BOPTA $[14,18]$. However, Gd-EOB-DTPA provides a stronger late hepatic and biliary enhancement, due to its elimination profile of $50 \%$ through the biliary pathway $[14,20]$.

Assessment of the biliary tree is also possible with these contrast agents by performing a T1-w cholangiography in the hepatobiliary phase $[18,20]$. Future applications of hepato-specific agents may include fibrosis staging and quantification of liver function [19,20].

Some protocol adaptions need to be addressed when using these agents. In the case of Gd-BOPTA, it implies that the patient has to be scanned twice, while for Gd-EOB-DTPA, the hepatobiliary phase can be obtained in the same examination [10]. Since a 10-20 min gap between the initial dynamic imaging and the hepatobiliary phase still exists in this case, imaging time can be filled with sequences unaffected by the presence of the contrast agent, such as T2-w FS and DWI [1,14,19]. GRE in and out of phase images and MRCP should, on the other hand, be performed before contrast administration [4,10,14]. Regarding the hepatobiliary phase, an increase in the flip angle to $30-35^{\circ}$ increases the signal of the enhancing liver and biliary tree and reduces the signal of non-enhancing structures, improving the conspicuity of hypointense lesions $[1,19]$.

\section{Liver MR at $1.5 \mathrm{~T}$ and $3 \mathrm{~T}$}

Scanners operating at a 3T magnetic field have been introduced and rapidly evolved over the last decade. These scanners take advantage of the increased SNR provided by $3 \mathrm{~T}$ fields relative to $1.5 \mathrm{~T}$, which is used to improve spatial resolution, to lower the acquisition time or a combination of the two $[8,11,17]$.

In most tissues, $\mathrm{T} 1$ relaxation times are generally longer at $3 \mathrm{~T}$ compared to $1.5 \mathrm{~T}$, while $\mathrm{T} 2$ relaxation times are almost unaffected. There is also greater fat and water spectral separation, which can lead to better fat suppression $[8,21]$.

The longer T1 relaxation times at 3T require longer TRs in T1-w sequences, with a consequent increase in acquisition time, in order to be equivalent to $1.5 \mathrm{~T}$ sequences [9]. Acquisition time is however limited by the breath-hold technique. An inversion recovery preparatory pulse can be added to increase $\mathrm{T} 1$ contrast or parallel imaging can be employed to reduce acquisition time [8].

Echo sampling for in-phase and out-phase imaging also suffers changes, since the precession frequencies of water and fat are reduce to half at 3T. With this magnetic field strength, the shortest out-phase and in-phase TEs are $1.15 \mathrm{msec}$ and $2.3 \mathrm{msec}$, respectively [8,9]. T2 sequences are generally acquired with a shorter TE to compensate for the greater T2* decay at 3T [9].

The dynamic study is one of the MR parameters that draw greatest benefits from a higher field strength. This is due to the substantial increase in liver tissue $\mathrm{T} 1$ relaxation times but minimal changes for $\mathrm{T} 1$ Gd shortening effects, leading to a higher liver-to-lesion contrast [17]. Higher SNR can be used to improve image resolution, using thinner slices, or reduce scan time, which is important for difficult patients [8]. The quality of the dynamic study can be further improved by a Dixon method of fat suppression [21]. Regarding DWI, there can be an up to two-fold increase in SNR [11,12].

Nevertheless, performing exams with a $3 \mathrm{~T}$ magnetic field also has disadvantages [17]. Firstly, regarding patient preparation, 3T compatibility of medical devices should be interrogated, since $1.5 \mathrm{~T}$ compatibility is not sufficient [8].

There is greater radiofrequency power deposition to the patient at $3 \mathrm{~T}$ relatively to $1.5 \mathrm{~T}$, especially in T2-w FSE sequences due to the larger number of radiofrequency pulses [21]. Energy deposition can be reduced by increasing the TR, reducing transmitter bandwidth, reducing the anatomical coverage, decreasing flip angles, using radiofrequency refocusing techniques or parallel imaging [8,9,21].

The higher field strength leads to greater magnetic susceptibility artefacts, with image distortion and signal loss but also greater conspicuity of metal or gas $[8,9,11]$.

Standing wave or dielectric artefact becomes a concern at 3T, with interactions between radiofrequency waves and the patient's body leading to heterogeneous deposition and large variations in signal $[8,17,21]$. FSE sequences, like T2-w sequences, are more affected, demonstrating areas of signal drop, especially in the left liver lobe, particularly in larger patients and in the presence of ascites (Fig. 6). Although abdominal pads between the patient's body and the coil may partially reduce these artefacts, they are now more consistently prevented by hardware improvements like dual source parallel radiofrequency transmission $[8,9,21]$. Alternatively, obese patients or patients with known ascites should preferably undergo 1.5T MR [21].

Chemical shift misregistration artefact occurring in fat-water interfaces also increases with the increasing field strength. It can be reduced by decreasing the receiver bandwidth, swapping frequency and phase encoding directions, and is eliminated with the use of fat suppression [4].

Greater sensitivity to susceptibility artefacts and field inhomogeneity lead to higher distortions in EPI sequences used in DWI and incomplete fat saturation. There are also reported significant differences between ADC measurements performed at 1.5 and 3T [12]. Some of these limitations may be reduced by the use of parallel imaging [8]. 


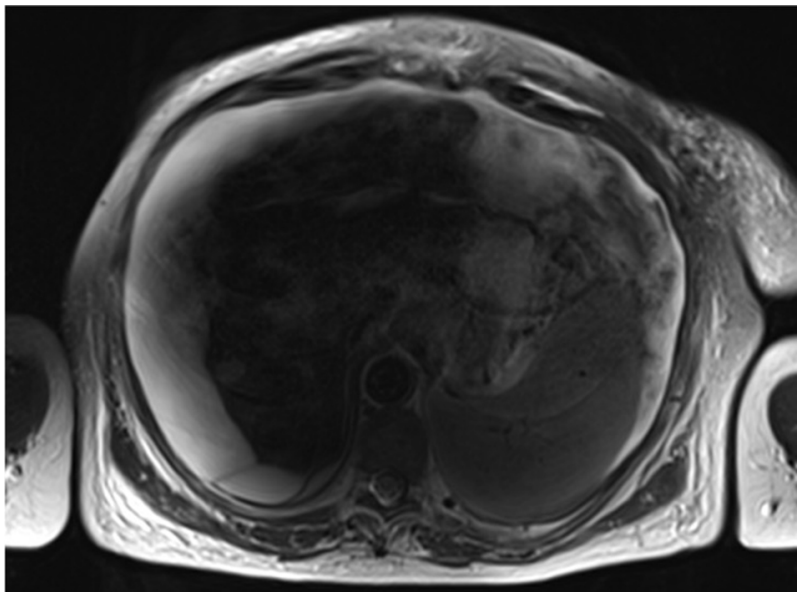

Fig. 6. Example of dieletric artefact in a T2-w FS sequence obtained with a 3T magnetic field in an obese patient with ascites, with dramatic signal drop in the center of the image.

\section{Advanced liver MR protocols}

\subsection{Perfusion $M R$}

In recent years there has been an increasingly wide use and development of vascular targeting agents, such as antiangiogenic drugs and vascular disruption agents (VDAs), for clinical use in the treatment of cancer as well as in clinical trials [22-25]. Considering the cytostatic nature of vascular targeting agents it has been suggested that the effect of vascular targeting therapies may be better assessed by evaluating the functional changes in tumour tissue than by observing the changes in tumour size alone $[22,26]$. Assuming this premise, multiparametric MR imaging can be a promising tool. In particular, DCE-MR has seen increased use in clinical trials of several antiangiogenic drugs and VDAs, as it can be used to investigate microvascular structures and their function, both noninvasively and quantitatively [22,23].

DCE-MR images are obtained by the venous injection of a low molecular weight Gd chelate at a constant rate. Within the tumour tissue, these agents extravasate from intravascular to the extravascular extracellular space (EES), which results in T1-w signal increase [27,28]. The rate of contrast agent extravasation to EES in the tumour tissue is determined by vessel leakiness and blood flow. Therefore, the signal measured in DCE-MR imaging represents a combination of permeability and perfusion [27]. DCE-MR is sensitive to alterations in vascular permeability, extracellular space, and blood flow. To ideally record the signal change in the supplying blood vessel and within the tumour, a fast injection rate of the contrast agent captured with high temporal resolution is required $[27,29]$. This signal enhancement of liver perfusion can be quantified either using a semi-quantitative or quantitative analysis. The semi-quantitative analysis is based on the calculation of parameters that can be extracted from time-signal intensity curves. The quantitative analysis needs computational-based curve cutting algorithms using a bi-compartmental model with arterial input function [27]. The parameters from both analysis methods have been shown to present correlation with tumoural angiogenesis [29].

With the semi-quantitative analysis, different parameters that characterize the shape of the normalized signal intensity (SI)-time curve can be extracted: (1) area under curve (AUC): expresses the amount of enhancement over a defined period of time (usually from starting increment of the SI-time curve to 60 or $90 \mathrm{~s}$ ); (2) maximum of SI or peak enhancement ratio (SI maximum-SI baseline/SI baseline) of the enhancing curve; (3) wash-in slope: determines the velocity of enhancement. It is calculated as the maximum change in enhancement per unit time, usually from $20 \%$ to $80 \%$ range of the increment curve; and (4) mean transit time (MTT): represents the mean time for blood to perfuse a region of tissue and is affected by the blood volume and blood flow in the region under analysis $[27,28]$. Although widely used, the semiquantitative analysis is highly affected by the gain factor of the acquisition systems, contrast media volume and injection rate, because the true concentration of contrast agent in the tissues is not estimated. Thus, differences in temporal resolution and injection rates can easily change the shape of SI curves, making comparison and quantification difficult $[29,30]$, not allowing a true physiologic insight into the behaviour of tumour vessels. Quantitative analysis is based on modelling the concentration change of the contrast agent using pharmacokinetic modelling techniques. An initial conversion step of SI to concentration values is needed. Concentration $v s$ time curves are then fitted using a bi-compartmental model (vessels and EES) with two vascular inputs (aorta and portal vein) [28-30]. Thus, the following parameters can be extracted from a mathematical model: (1) Ktrans (forward volume transfer constant): determines the influx of the contrast agent from the intravascular space to the EES. It predominantly represents vascular permeability in a permeability-limited (high flow) situation, but also represents the blood flow into the tissue in a flow-limited situation; (2) Kep (reverse reflux rate constant): expresses the return process of the contrast agent from the EES to the intravascular space; and (3) Ve (volume fraction of EES): an indirect measure representing the cellular density of the tissue $[27,30]$. In comparison to the semiquantitative analysis, these parameters require additional calculations to generate parametric maps obtained after a pixel-by-pixel curve fitting process of the region under analysis, being more time consuming and computationally demanding. After generating parametric maps (Fig. 7), the mean or median values within region of interest are usually calculated to represent tumour microvasculature, but histogram analysis (Fig. 8) or heterogeneity in parametric maps may also provide additional information $[27,30]$. Throughout the years, numerous pharmacokinetic models have been proposed by Tofts et al., Brix et al. and Larsson et al. [31-33], using a single arterial input function [27]. Since hepatocellular carcinoma receives major blood supply from hepatic arteries, this single input model is frequently used in the literature. On the other hand, for parenchymal and metastatic disease, in which arterial and portal vein supply is present, the dual input one compartment model proposed by Materne et al. [34] is more appropriate.

Regarding technical details, the protocol includes a T1-w 3D spoiled GRE technique using variable flip angles. Compared with 2D imaging sequences, the 3D technique eliminates inaccuracies due to the radiofrequency excitation pulse profile, and also has the advantage of better SNR [30]. Adequate T1 mapping is necessary for quantitative analysis and this is usually achieved with different flip angles, as suggested by Wang et al. [35]. In order to track liver perfusion reliably, MRI of the liver should employ a high temporal resolution technique (i.e. repeated

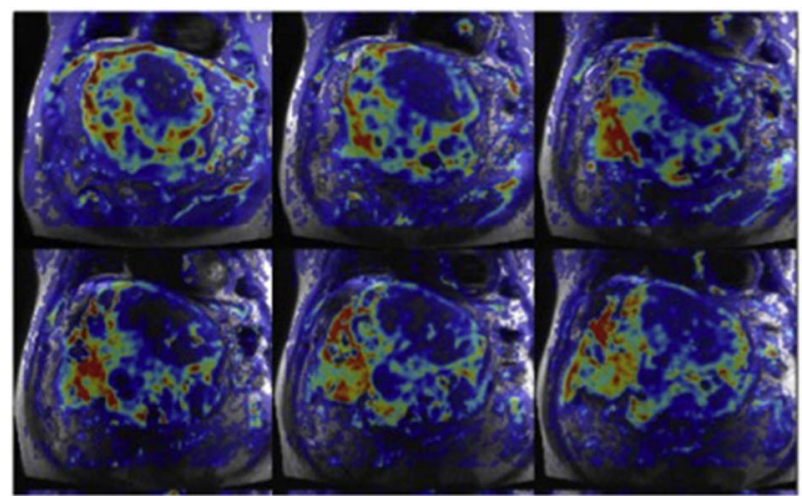

Fig. 7. Representative DCE-MR imaging parametric ktrans maps fused with $\mathrm{T} 2$ coronal images in a patient with advanced hepatocellular carcinoma. Red areas correspond to higher tumour vascularity and hence higher Ktrans values. (For interpretation of the references to colour in this figure legend, the reader is referred to the web version of this article.) 

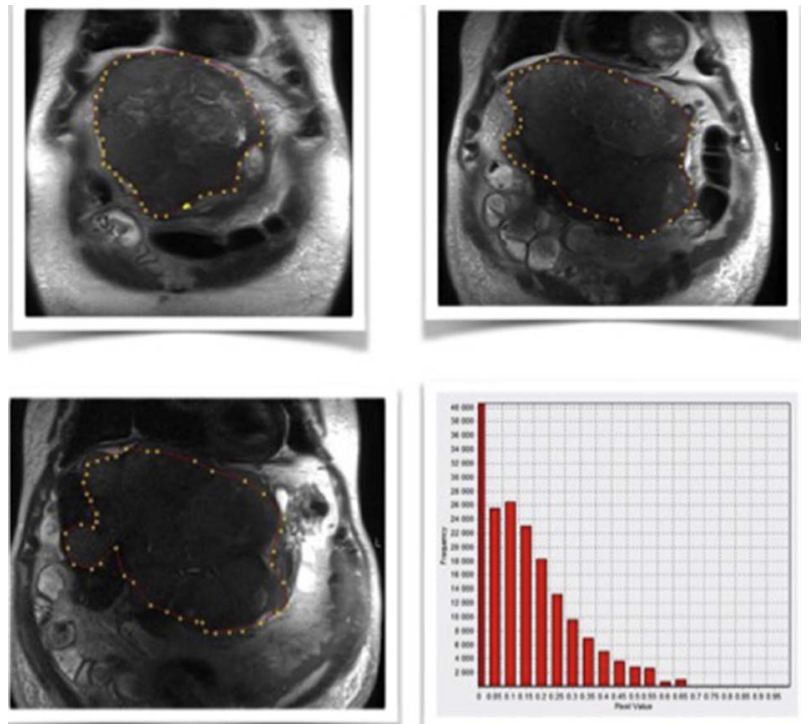

Fig. 8. Representative $R O I$ tracing in $\mathrm{T} 2$ image and corresponding total volume histogram analysis of the ktrans parameter in same patient as mentioned in Fig. 7.

Table 2

Illustrative example of a perfusion MRI sequence performed in a 1.5T scanner (adapted from Thng et al).

\begin{tabular}{ll}
\hline MRI platform & $1,5 \mathrm{~T}$ \\
\hline Type of pulse sequence & 3D FLASH \\
TR & $2.72 \mathrm{~ms}$ \\
TE & $1 \mathrm{~ms}$ \\
Partition thickness & $8 \mathrm{~mm}$ \\
Slices per slab & 10 \\
Matrix & $256 \times 169$ \\
Phase encode direction & Anterior to posterior \\
Number of averages & 1 \\
Sensitivity encoding & 2 \\
$\quad$ factor & $2^{\circ}$ and $14^{\circ}$ \\
Flip angle before contrast & $14^{\circ}$ \\
Flip angle after contrast & $490 \mathrm{Mhz}$ \\
Bandwidth & Yes \\
RF spoiling & $1.98 \mathrm{~s}$ per slab of 10 slices \\
Temporal resolution & 10 measurements of each flip angle averaged for \\
Precontrast scans & calculation of native T1 \\
& 0.2 mmol/kg at 3 mL/s followed by 20 mL flush \\
Gadolinium injection & Quiet breathing \\
Patient respiration & A total of 180 consecutive measurements. Inject \\
Post contrast scans & contrast only when the 20th measurement has been \\
& completed \\
Scan sections to use for & Center 6 image sections only \\
\multicolumn{1}{c}{ processing } & \\
\hline & \\
\hline
\end{tabular}

imaging of the same area in the liver about every 4 s), in a scan plane that shows the lesion or area of interest. Ideally, the aorta and the portal vein should be included in the same image sections. Hence, an oblique imaging plane afforded by the MRI technique can be particularly helpful to ensure all these structures are included [30]. The signal intensity changes within these structures, together with the T1 calibration maps, are used to derive the Gd contrast concentration-time curves in the liver and tumours. One of the challenges in acquiring high quality liver perfusion MRI is respiratory motion, which can substantially degrade image quality. Therefore, imaging in quiet superficial breathing has been suggested to minimize these effects. An example of a liver perfusion MR protocol for use in a commercial scanner is described in Table 2 [30].

\subsection{Quantitative MR for diffuse liver diseases: fat, iron and fibrosis}

Liver steatosis is the hallmark of non-alcoholic liver disease (NAFLD), but is also a common feature in several diffuse liver diseases. Liver iron concentration (LIC) is used as a surrogate of body iron stores in patients with primary and secondary hemochromatosis. Nevertheless, iron deposition is also present in different diffuse liver diseases. Chronic liver injury results in liver fibrosis and, consequently, can lead to liver cirrhosis. Because liver biopsy is invasive and has several limitations, advanced MR imaging techniques have emerged as non-invasive quantitative biomarkers for fat and iron deposits, and assessment of liver fibrosis.

\subsubsection{MR quantification of hepatic fat (proton density fat fraction)}

MR spectroscopy (MRS) is considered the imaging gold-standard for fat quantification. Knowing a priori the resonance frequencies of fat and water protons, their concentrations can be quantified directly from their spectral signal [36]. Fat (triglyceride) signal has multiple frequency components, the dominant one being located at a frequency shift of $420 \mathrm{~Hz}$ (1.46 ppm) relative to water peak, on 3T magnetic filed. Fat proton density results from the sum of these diverse multiple fat peaks. MRS is an accurate and reproducible method for quantification of proton density fat fraction (PDFF) [36]. However, it is time consuming and it is only available in specialized hospital or research centres.

Multi-echo chemical shift encoded (MECSE) GRE sequences, performed with more than 3 echoes (usually between 6 and 12), have recently emerged as an accurate tool for PDFF quantification [36,37] (Fig. 9). These sequences take advantage of the chemical shift of water and fat protons. For precise fat quantification, these sequences must be corrected for the main confounding factors (T1 bias, effect of $\mathrm{T} 2$ * relaxation, fat spectral complexity) [36,37]. Fat protons have a shorter T1 relaxation time than water protons, which may introduce a significant bias in fat fraction estimation if the acquisition is T1-w. This "T1 bias" can be minimized using a low flip angle $\left(<10^{\circ}\right)$ [38]. The fitting model should also incorporate the multiple frequencies of fat spectrum (multipeak fat spectral modelling) $[36,37,39]$. The effect of T2* decay between different echoes confounds PDFF quantification, mainly when concomitant iron deposition is present, but also in normal

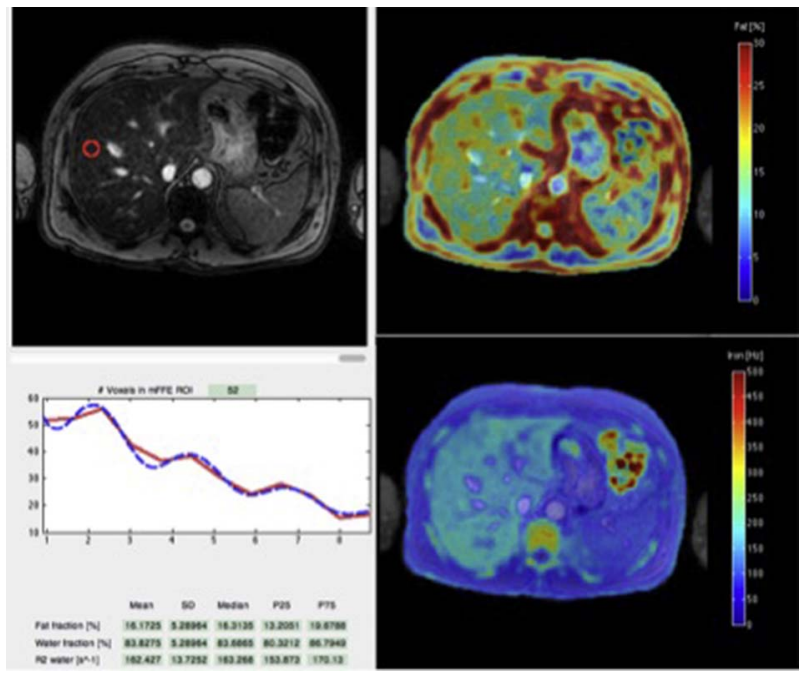

Fig. 9. Fat (PDFF) and iron (R2*) quantification using MECSE-MR sequence, in male patient with non-alcoholic fatty liver disease. (a) Magnitude images used for fat and iron quantification, and the respective signal decay curve calculated with QLiver software (QUIBIM SL, Valencia, Spain). A ROI area in the right hepatic lobe determined a $\mathrm{PDFF}=16 \%$ and $\mathrm{R} 2 *=162 \mathrm{~s}-1$. Steatosis and iron overload frequently co-exist in diffuse liver diseases. (b) Parametric maps of PDFF (c) and iron related-R2* (d) measurements are useful to demonstrate the distribution and the amount of fat and iron throughout the liver parenchyma. 
livers. The signal fitting model of the multiple echoes allows to quantify fat and to estimate the T2* decay. The T2* estimation is used to correct the effect of T2* in PDFF quantification. Moreover, because liver T2* is related to the amount of iron deposition, it can also be used for simultaneous quantification of iron deposits [36,40-42]. Nevertheless, because large iron content lead to extremely low signal in GRE sequences, PDFF measurements may be corrupted in livers with severe iron overload [41].

Fat measurements can be performed in parametric maps, which have the advantage to demonstrate not only the amount but also the distribution of fat throughout the liver parenchyma (Fig. 9). PDFF quantification can be performed with commercially available tools (IDEAL-IQ ${ }^{\circ}$, GE; mDIXON Quant ${ }^{\circ}$, PHILIPS), or using in-house developed software. MECSE-MR imaging sequences are accurate for quantification of hepatic steatosis $[41,43,44]$, being repeatable [45] and reproducible across different MR scanner platforms at $1.5 \mathrm{~T}$ and $3 \mathrm{~T}$ scanners [46].

\subsubsection{MR quantification of hepatic iron overload}

The most traditionally used method for quantification of liver iron concentration (LIC) uses 5 different IP GRE sequences and compares the signal intensity between the liver and a non-overloaded reference tissue (paraspinal muscles) [47]. However, these ratio measurements are biased by coexisting hepatic steatosis and/or muscle fatty infiltration. Furthermore, this method is not compatible with phase-array coils and is not calibrated for $3 \mathrm{~T}$ equipment.

Relaxometry methods, on the other hand, use a series of images acquired with increasing TE, either with a SE or a multi-echo GRE sequence, and they can be performed with surface coils. The liver signal intensity is modelled as a function of TE and fitted to either a monoexponential or bi-exponential decay model, calculating signal decay constants (T2 or T2*) or signal decay rates (R2 or R2*) [48]. Liver T2 and T2* (or R2, R2*) are closely related to LIC, and can be used in clinical practice as surrogates of LIC, provided that they are calculated with validated acquisition and analysis protocols [49].

A R2 relaxometry method [50] is currently available as a commercial service (FerriScan ${ }^{\circ}$ ). Five T2-w sequences are acquired during freebreathing, and images are forwarded for centralized image data analysis and R2 measurements. R2 techniques are less sensitive to external magnetic inhomogeneities [48] but require a longer acquisition time than R2* techniques, being more prone to motion artefacts.

$\mathrm{R} 2 *$ relaxometry methods are usually performed with SPGR multiecho sequences, during one or two breath-hold acquisitions [42,48,51]. T2* or R2* measurements can be calculated using commercial postprocessing software (CMR Tools ${ }^{\circ}$ ) or in-house developed software. The first echo should be as short as possible (1 ms or less) and the echo spacing should be short enough (approximately $1 \mathrm{~ms}$ or less) [42], to guarantee that the signal decay is captured even in high iron-loaded livers. The LIC ( $\mathrm{mg} \mathrm{Fe} / \mathrm{g}$ or $\mu \mathrm{mol} \mathrm{Fe} / \mathrm{g}$ ) can be estimated from the R2*/ T2* measurements, using calibration curves that were pre-validated against liver biopsies [42,48,51,52]. As mentioned above, MECSE-MR sequences, the same as described for fat quantification, can also be used for R2* estimation and LIC quantification (Figs. 9 and 10) [40-42,48] while simultaneously assessing the liver fat content (Figs. 9 and 10). Nevertheless, relaxation rates are dependent on magnetic field strength and imaging acquisition parameters, and R2* relaxometry still needs technical standardization and consensus [42].

\subsubsection{MR quantification of hepatic fibrosis}

MR elastography (MRE) evaluates liver stiffness, measuring the speed of shear waves propagating through the liver, which are induced by a vibrating compression device [53]. Then, using a phase-contrast MR sequence with motion-encoding gradients, these shear waves are detected and converted in quantitative maps (elastograms) of tissue stiffness (measured in $\mathrm{kPa}$ ). MRE can be implemented on a conventional MR system, using modified GRE or SE sequences and adding
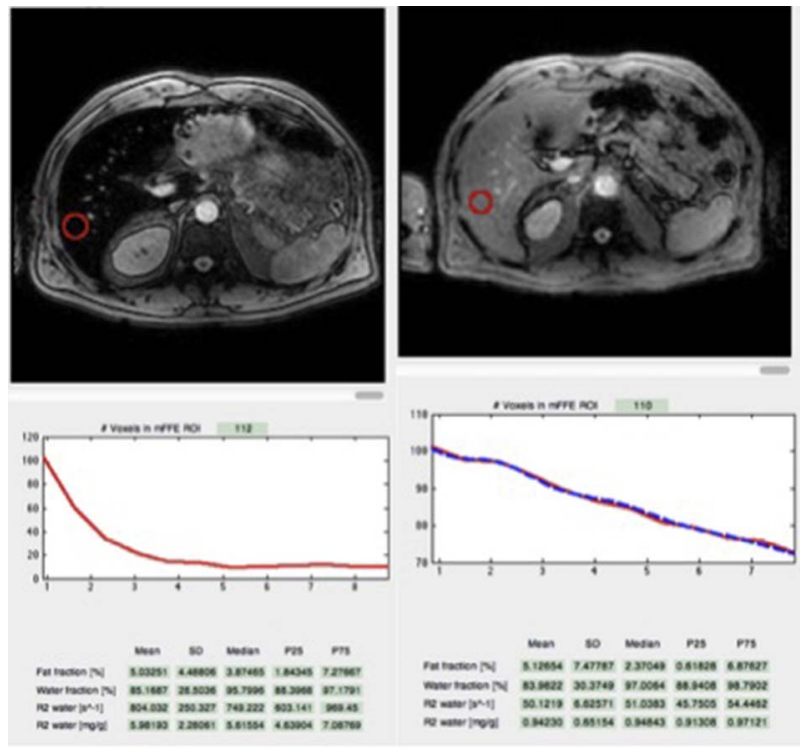

Fig. 10. Iron (R2*) quantification using MECSE-MR sequence, in male patient with hereditary hemochromatosis, in two different time points. Magnitude images from a MECSE MR sequence acquired with 12 different TEs, used for R2* quantification, and the respective signal decay curve, calculated with QLiver" software (QUIBIM SL, Valencia, Spain). (a) MR examination in 2013 demonstrates low signal intensity of liver parenchyma, which is related to severe iron overload. A plot of mean signal intensity within a circular ROI is modeled as a function of TE, as a bi-exponential decay curve. Estimated R2* is $804 \mathrm{~s}-1$. (b) In 2015, after intensive treatment with phlebotomies and removal of $3,85 \mathrm{~g}$ total body iron stores, the liver R2* is $51 \mathrm{~s}-1$. These measurements can be used for follow up of patients with iron overload disorders.

hardware to generate the waves and software for acquisition and processing. Liver stiffness is directly related to the stage of fibrosis, increasing with the progression of the disease [53,54]. MRE has a high sensitivity and specificity in diagnosing and staging of liver fibrosis and it is now considered as the best non-invasive tool to assess liver fibrosis [53]. Nevertheless, besides fibrosis, hepatic stiffness also increases with liver inflammation. Furthermore, MRE is impaired in patients with moderate to severe iron overload [55], but this limitation might be overcome by using modified SE techniques [56]. In comparison with ultrasound elastography methods, MRE has the advantage to analyse a larger volume of liver parenchyma, evaluating the whole liver, and not be limited by obesity or ascites [53].

A T1 mapping technique has also been recently purposed for the evaluation and scoring of liver fibrosis [57]. T1 relaxation time map is obtained using a single breath-hold Shortened Modified Look Locker Inversion recovery (shMOLLI) sequence, in the axial plane through the right lobe of the liver and the spleen [57]. T1 values need to be adjusted for the hepatic iron level, because iron accumulation in the presence of fibrosis can mimic normal $\mathrm{T} 1$ values. This method has demonstrated a good correlation with histological stage of liver fibrosis and it seems promising in predicting negative clinical outcomes in patients with chronic liver disease [58].

DWI and intra-voxel incoherent motion (IVIM) DWI MR imaging have also been proposed for assessment of liver fibrosis: livers with higher fibrosis stages would have lower ADC values and lower perfusion related IVIM parameters than livers without fibrosis [59]. However, ADC and IVIM measurements are not accurate enough to be used in clinical practice for staging liver fibrosis, in diffuse liver diseases [60]. Also, its use has been limited by the large variability in the cut-off values and by the possible confounding effect of hepatic steatosis $[60,61]$ or iron overload [61].

\section{Conflicts of interest}

The authors have no conflicts of interest to disclose. 


\section{References}

[1] Y.L. Thian, A.M. Riddell, D.M. Koh, Liver-specific agents for contrast-enhanced MRI: role in oncological imaging, Cancer Imag. 13 (2013) 567-579, http://dx.doi. org/10.1102/1470-7330.2013.0050.

[2] A.P. Matos, F. Velloni, M. Ramalho, M. Alobaidy, A. Rajapaksha, R.C. Semelka, Focal liver lesions: practical magnetic resonance imaging approach, World J. Hepatol. 7 (2015) 1987-2008, http://dx.doi.org/10.4254/wjh.v7.i16.1987.

[3] B.E. Van Beers, J.-L. Daire, P. Garteiser, New imaging techniques for liver diseases, J. Hepatol. 62 (2015) 690-700, http://dx.doi.org/10.1016/j.jhep.2014.10.014.

[4] F.F. Guglielmo, D.G. Mitchell, C.G. Roth, S. Deshmukh, Hepatic MR imaging techniques, optimization, and artifacts, Magn. Reson. Imag. Clin. N. Am. 22 (2014) 263-282, http://dx.doi.org/10.1016/j.mric.2014.04.004.

[5] E.K. O'Neill, J.R. Cogley, F.H. Miller, The ins and outs of liver imaging, Clin. Liver Dis. 19 (2015) 99-121, http://dx.doi.org/10.1016/j.cld.2014.09.006.

[6] M. Taupitz, P. Asbach, A.E. Mahfouz, B. Hamm, The liver, in: B. Hamm, G.P. Krestin, M. Laniado, V. Nicolas, M. Taupitz (Eds.), MR Imaging of the Abdomen and Pelvis, Thieme, New York, 2009, pp. 1-46.

[7] G.E. Wile, J.R. Leyendecker, Magnetic resonance imaging of the liver: sequence optimization and artifacts, Magn. Reson. Imag. Clin. N. Am. 18 (2010) 525-547, http://dx.doi.org/10.1016/j.mric.2010.07.010.

[8] K.J. Chang, I.R. Kamel, K.J. Macura, D.A. Bluemke, 3.0-T MR imaging of the abdomen: comparison with 1.5T, Radiographics 28 (2008) 1983-1998, http://dx. doi.org/10.1148/rg.287075154.

[9] M. Ramalho, E. Altun, V. Herédia, M. Zapparoli, R. Semelka, Liver MR imaging: 1.5T versus 3T, Magn. Reson. Imag. Clin. N. Am. 15 (2007) 321-347, http://dx.doi. org/10.1016/j.mric.2007.06.003.

[10] C. LeBedis, A. Luna, J.A. Soto, Use of magnetic resonance imaging contrast agents in the liver and biliary tract, Magn. Reson. Imag. Clin. N. Am. 20 (2012) 716-737, http://dx.doi.org/10.1016/j.mric.2012.07.006.

[11] G.S. Chilla, C.H. Tan, C. Xu, C.L. Poh, Diffusion weighted magnetic resonance imaging and its recent trend-a survey, Quant. Imag. Med. Surg. 5 (2015) 407-422, http://dx.doi.org/10.3978/j.issn.2223-4292.2015.03.01.

[12] S. Lewis, H. Dyvorne, Y. Cui, B. Taouli, Diffusion-weighted imaging of the liver: techniques and applications, Magn. Reson. Imag. Clin. N. Am. 22 (2014) 373-395, http://dx.doi.org/10.1016/j.mric.2014.04.009.

[13] G.E. Boyle, M. Ahern, J. Cooke, N.P. Sheehy, J.F. Meaney, An interactive taxonomy of MR imaging sequences, Radiographics 26 (2006) e24, http://dx.doi.org/10. 1148/rg.e24.

[14] N. Albiin, MRI of focal liver lesions, Curr. Med. Imag. Rev. 8 (2012) 107-116, http://dx.doi.org/10.2174/157340512800672216.

[15] M.M. Jafar, A. Parsai, M.E. Miquel, Diffusion-weighted magnetic resonance imaging in cancer: Reported apparent diffusion coefficients, in-vitro and in-vivo reproducibility, World J Radiol. 8 (2016) 21-49. 10.4329/wjr.v8.i1.21.

[16] M.R. Bashir, Magnetic resonance contrast agents for liver imaging, Magn. Reson. Imaging Clin. N. Am. 22 (2014) 283-293, http://dx.doi.org/10.1016/j.mric.2014 04.002 .

[17] J. Huh, S.Y. Kim, B.M. Yeh, S.S. Lee, K.W. Kim, E.H. Wu, Z. Jane Wang, L.Q. Zhao, W.C. Chang, Troubleshooting arterial-phase MR images of gadoxetate disodiumenhanced liver, Korean J. Radiol. 16 (2015) 1207-1215, http://dx.doi.org/10. 3348/kjr.2015.16.6.1207.

[18] E. Neri, M.A. Bali, A. Ba-Ssalamah, P. Boraschi, G. Brancatelli, F.C. Alves, L. Grazioli, T. Helmberger, J.M. Lee, R. Manfredi, L. Martì-Bonmatì, C. Matos, E.M. Merkle, B. Op De Beeck, W. Schima, S. Skehan, V. Vilgrain, C. Zech, C. Bartolozzi, ESGAR consensus statement on liver MR imaging and clinical use of liver-specific contrast agents, Eur. Radiol. 26 (2016) 921-931, http://dx.doi.org/ 10.1007/s00330-015-3900-3.

[19] K. Jhaveri, S. Cleary, P. Audet, F. Balaa, D. Bhayana, K. Burak, S. Chang, E. Dixon, M. Haider, M. Molinari, C. Reinhold, M. Sherman, Consensus statements from a multidisciplinary expert panel on the utilization and application of a liver-specific MRI Contrast agent (gadoxetic acid), Am. J. Roentgenol. 204 (2015) 498-509, http://dx.doi.org/10.2214/AJR.13.12399.

[20] C. Burke, L. Alexander Grant, V. Goh, N. Griffin, The role of hepatocyte-specific contrast agents in hepatobiliary magnetic resonance imaging, Semin. Ultrasound CT MRI 34 (2013) 44-53, http://dx.doi.org/10.1053/j.sult.2012.11.004.

[21] R. Girometti, 3.0 Tesla magnetic resonance imaging: A new standard in liver imaging?, World J. Hepatol. 7 (2015) 1894. doi: 10.4254/wjh.v7. i15.1894.

[22] I. Joo, J.M. Lee, R. Grimm, J.K. Han, B.I. Choi, Monitoring vascular disrupting therapy in a rabbit liver model: relationship between tumor perfusion parameters at IVIM diffusion-weighted MR imaging and those at dynamic contrast-enhanced MR Imaging, Radiology 278 (2016) 104-113, http://dx.doi.org/10.1148/radiol. 2015141974.

[23] M.J. McKeage, B.C. Baguley, Disrupting established tumor blood vessels: an emerging therapeutic strategy for cancer, Cancer 116 (2010) 1859-1871, http://dx. doi.org/10.1002/cncr.24975.

[24] B.A. Salmon, H.W. Salmon, D.W. Siemann, Monitoring the treatment efficacy of the vascular disrupting agent CA4P, Eur. J. Cancer 43 (2007) 1622-1629, http://dx. doi.org/10.1016/j.ejca.2007.03.018.

[25] J.C. Miller, H.H. Pien, D. Sahani, A.G. Sorensen, J.H. Thrall, Imaging angiogenesis: applications and potential for drug development, J. Natl. Cancer Inst. 97 (2005) 172-187, http://dx.doi.org/10.1093/jnci/dji023.

[26] V. Goh, S. Halligan, C.I. Bartram, Quantitative tumor perfusion assessment with multide- tector CT: are measurements from two commercial software packages interchangeable? Radiology 242 (2007) 777-782, http://dx.doi.org/10.1148/ radiol. 2423060279 .
[27] B.B. Chen, T.T. Shih, DCE-MRI in hepatocellular carcinoma- clinical and therapeutic image biomarker, World J. Gastroenterol. 20 (2014) 3125-3134, http://dx.doi.org/ 10.3748/wjg.v20.i12.3125.

[28] G. Brix, J. Griebel, F. Kiessling, F. Wenz, Tracer kinetic modelling of tumour angiogenesis based on dynamic contrast- enhanced CT and MRI measurements, Eur. J. Nucl. Med. Mol. Imag. 37 (Suppl. 1) (2010) S30-S51, http://dx.doi.org/10.1007/ s00259-010-1448-7.

[29] S. Sourbron, Technical aspects of MR perfusion, Eur. J. Radiol. 76 (2010) 304-313, http://dx.doi.org/10.1016/j.ejrad.2010.02.017.

[30] C.H. Thng, T.S. Koh, D.J. Collins, D.M. Koh, Perfusion magnetic resonance imaging of the liver, World J. Gastroenterol. 16 (2010) 1598-1609.

[31] P.S. Tofts, D.A. Wicks, G.J. Barker, The MRI measurement of NMR and physiological parameters in tissue to study disease process, Prog. Clin. Biol. Res. 363 (1991) 313-325.

[32] G. Brix, W. Semmler, R. Port, L.R. Schad, G. Layer, W.J. Lorenz, Pharmacokinetic parameters in CNS Gd-DTPA enhanced MR imaging, J. Comput. Assist. Tomogr. 15 (1991) 621-628.

[33] H.B. Larsson, M. Stubgaard, J.L. Frederiksen, M. Jensen, O. Henriksen, O.B. Paulson, Quantitation of blood-brain barrier defect by magnetic resonance imaging and gadolinium-DTPA in patients with multiple sclerosis and brain tumors, Magn. Reson. Med. 16 (1990) 117-131.

[34] R. Materne, A.M. Smith, F. Peeters, J.P. Dehoux, A. Keyeux, Y. Horsmans, B.E. Van Beers, Assessment of hepatic perfusion parameters with dynamic MRI, Magn. Reson. Med. 47 (2002) 135-142.

[35] H.Z. Wang, S.J. Riederer, J.N. Lee, Optimizing the precision in T1 relaxation estimation using limited flip angles, Magn. Reson. Med. 5 (1987) 399-416.

[36] S.B. Reeder, I. Cruite, G. Hamilton, C.B. Sirlin, Quantitative assessment of liver fat with magnetic resonance imaging and spectroscopy, J. Magn. Reson. Imag. 34 (2011), http://dx.doi.org/10.1002/jmri.22775 (spcone).

[37] S.B. Reeder, H.H. Hu, C.B. Sirlin, Proton density fat-fraction: a standardized MR based biomarker of tissue fat concentration, J. Magn. Reson. Imag. 36 (2012) 1011-1014, http://dx.doi.org/10.1002/jmri.23741.

[38] C.Y. Liu, C.A. McKenzie, H. Yu, J.H. Brittain, S.B. Reeder, Fat quantification with IDEAL gradient echo imaging: correction of bias from T1 and noise, Magn. Reson. Med. 58 (2007) 354-364, http://dx.doi.org/10.1002/mrm.21301.

[39] X. Wang, D. Hernando, S.B. Reeder, Sensitivity of chemical shift-encoded fat quantification to calibration of fat MR spectrum, Magn. Reson. Med. 75 (2015) 845-851, http://dx.doi.org/10.1002/mrm.25681.

[40] L. Martí-Bonmatí, A. Alberich-Bayarri, J. Sánchez-González, Overload hepatitides: quanti-qualitative analysis, Abdom. Imag. 37 (2011) 180-187, http://dx.doi.org/ 10.1007/s00261-011-9762-5.

[41] M. França, A. Alberich-Bayarri, L. Martí-Bonmatí, Accurate simultaneous quantification of liver steatosis and iron overload in diffuse liver diseases with MRI, Abdom Radiol. (2017), http://dx.doi.org/10.1007/s00261-017-1048-0.

[42] T. Yokoo, J.D. Browning, Fat and iron quantification in the liver: past, present, and future, Top. Magn. Reson. Imag. 23 (2014) 73-94, http://dx.doi.org/10.1097/ RMR.0000000000000016.

[43] A. Tang, J. Tan, M. Sun, G. Hamilton, M. Bydder, T. Wolfson, A.C. Gamst, M. Middleton, E.M. Brunt, R. Loomba, J.E. Lavine, J.B. Schwimmer, C.B. Sirlin, Nonalcoholic fatty liver disease: MR imaging of liver proton density fat fraction to assess hepatic steatosis, Radiology 267 (2013) 422-431, http://dx.doi.org/10. 1148/radiol.12120896.

[44] I.S. Idilman, H. Aniktar, R. Idilman, G. Kabacam, B. Savas, A. Elhan, A. Celik, K. Bahar, M. Karcaaltincaba, Hepatic steatosis quantification by proton density fat fraction with MR imaging versus liver biopsy, Radiology 267 (2013) 767-775, http://dx.doi.org/10.1148/radiol.13121360.

[45] A. Tyagi, O. Yeganeh, Y. Levin, J.C. Hooker, G.C. Hamilton, T. Wolfson, A. Gamst, A.K. Zand, E. Heba, R. Loomba, J. Schwimmer, M.S. Middleton, C.B. Sirlin, Intraand inter-examination repeatability of magnetic resonance spectroscopy, magnitude-based MRI, and complex-based MRI for estimation of hepatic proton density fat fraction in overweight and obese children and adults, Abdom. Imag. 40 (2015) 3070-3077, http://dx.doi.org/10.1007/s00261-015-0542-5.

[46] G.H. Kang, I. Cruite, M. Shiehmorteza, T. Wolfson, A.C. Gamst, G. Hamilton, M. Bydder, M.S. Middleton, C.B. Sirlin, Reproducibility of MRI-determined proton density fat fraction across two different MR scanner platforms, J. Magn. Reson. Imag. 34 (2011) 928-934, http://dx.doi.org/10.1002/jmri.22701.

[47] Y. Gandon, D. Olivié, D. Guyader, C. Aubé, F. Oberti, V. Sebille, Y. Deugnier, Noninvasive assessment of hepatic iron stores by MRI, Lancet 363 (2004) 357-362, http://dx.doi.org/10.1016/S0140-6736(04)15436-6.

[48] C.B. Sirlin, S.B. Reeder, Magnetic resonance imaging quantification of liver iron, Magn. Reson. Imag. Clin. N. Am. 18 (2010) 359-381, http://dx.doi.org/10.1016/j. mric.2010.08.014.

[49] J.C. Wood, Use of magnetic resonance imaging to monitor iron overload, Hematol Oncol. Clin. N. Am. 28 (2014) 747-764, http://dx.doi.org/10.1016/j.hoc.2014.04. 002.

[50] T.G. St Pierre, P.R. Clark, W. Chua-anusorn, A.J. Fleming, G.P. Jeffrey, J.K. Olynyk, P. Pootrakul, E. Robins, R. Lindeman, Noninvasive measurement and imaging of liver iron concentrations using proton magnetic resonance, Blood 105 (2005) 855-861, http://dx.doi.org/10.1182/blood-2004-01-0177.

[51] J.C. Wood, MRI R2 and R2* mapping accurately estimates hepatic iron concentration in transfusion-dependent thalassemia and sickle cell disease patients, Blood 106 (2005) 1460-1465, http://dx.doi.org/10.1182/blood-2004-10-3982.

[52] M.R. Garbowski, J.P. Carpenter, G. Smith, M. Roughton, M.H. Alam, T. He, D.J. Pennell, J.B. Porter, Biopsy-based calibration of T2* magnetic resonance for estimation of liver iron concentration and comparison with R2 Ferriscan, J. Cardiovasc. Magn. Reson. 16 (2014) 1-11, http://dx.doi.org/10.1186/1532-429X- 


$$
\text { 16-40. }
$$

[53] E.M. Godfrey, L. Mannelli, N. Griffin, D.J. Lomas, Magnetic resonance elastography in the diagnosis of hepatic fibrosis, Semin. Ultrasound CT MR 34 (2013) 81-88, http://dx.doi.org/10.1053/j.sult.2012.11.007.

[54] K.J. Glaser, A. Manduca, R.L. Ehman, Review of MR elastography applications and recent developments, J. Magn. Reson. Imag. 36 (2012) 757-774, http://dx.doi.org/ 10.1002/jmri.23597.

[55] S.K. Venkatesh, M. Yin, R.L. Ehman, Magnetic resonance elastography of liver: technique, analysis, and clinical applications, J. Magn. Reson. Imag. 37 (2013) 544-555, http://dx.doi.org/10.1002/jmri.23731.

[56] Y.K. Mariappan, B. Dzyubak, K.J. Glaser, S.K. Venkatesh, C.B. Sirlin, J. Hooker, K.P. McGee, R.L. Ehman, Application of modified spin-echo-based sequences for hepatic MR elastography: evaluation, comparison with the conventional gradientecho sequence, and preliminary clinical experience, Radiology 282 (2016) 390-398, http://dx.doi.org/10.1148/radiol.2016160153.

[57] R. Banerjee, M. Pavlides, E.M. Tunnicliffe, S.K. Piechnik, N. Sarania, R. Philips, J.D. Collier, J.C. Booth, J.E. Schneider, L.M. Wang, D.W. Delaney, K.A. Fleming, M.D. Robson, E. Barnes, S. Neubauer, Multiparametric magnetic resonance for the non-invasive diagnosis of liver disease, J. Hepatol. 60 (2014) 69-77, http://dx.doi. org/10.1016/j.jhep.2013.09.002.

[58] M. Pavlides, R. Banerjee, J. Sellwood, C.J. Kelly, M.D. Robson, J.C. Booth, J. Collier, S. Neubauer, E. Barnes, Multiparametric magnetic resonance imaging predicts clinical outcomes in patients with chronic liver disease, J. Hepatol. 64 (2016) 308-315, http://dx.doi.org/10.1016/j.jhep.2015.10.009.

[59] B. Taouli, D.M. Koh, Diffusion-weighted MR imaging of the liver, Radiology 254 (2010) 47-66, http://dx.doi.org/10.1148/radiol.09090021.

[60] M. França, L. Martí-Bonmatí, A. Alberich-Bayarri, P. Oliveira, S. Guimaraes, J. Oliveira, J. Amorim, J.S. Gonzalez, J.R. Vizcaíno, H.P. Miranda, Evaluation of fibrosis and inflammation in diffuse liver diseases using intravoxel incoherent motion diffusion-weighted MR imaging, Abdom. Radiol. (2016), http://dx.doi.org/ 10.1007/s00261-016-0899-0.

[61] H.S. Leitão, S. Doblas, G. d'Assignies, P. Garteiser, J.L. Daire, V. Paradis, C.F. Geraldes, V. Vilgrain, B.E. Van Beers, Fat deposition decreases diffusion parameters at MRI: a study in phantoms and patients with liver steatosis, Eur. Radiol. 23 (2012) 461-7. doi:10.1007/s00330-012-2626-8. 